\title{
Reassembling embryos in vitro from component stem cells
}

\author{
Cell Research (2017) 27:961-962. doi:10.1038/cr.2017.61; published online 21 April 2017
}

\begin{abstract}
Researchers at the University of Cambridge, UK have succeeded in reconstructing mouse embryos by combining pluripotent embryonic and multipotent trophoblast stem cells in a 3D scaffold; the study from the laboratory of Professor ZernickaGoetz, recently published in Science, provides a break-through tool to probe early mammalian development outside the uterus. Achieving a similar feat with human cells might necessitate reconsideration of the 14-day rule as a limitation of such research.
\end{abstract}

In the short period between fertilization and implantation into the mother's uterus, the fertilized egg develops into the blastocyst, a spherical structure comprising cells of the embryonic and extra-embryonic lineages. Inside the blastocyst, the founding tissue of the embryo proper (epiblast) is lined by the primitive endoderm, which later becomes the yolk sac. Both are enveloped by the trophectoderm, which mediates implantation and harbors the precursor cells of the placenta. These lineages can be captured permanently in vitro as self-renewing stem cells, recapitulating the developmental potential of their tissues of origin. Cultured epiblast cells give rise to pluripotent embryonic stem cells (ESCs), while multipotent trophoblast stem cells (TSCs) can be derived from the polar trophectoderm $[1,2]$ (Figure 1).

While preimplantation stages are well understood, studies of the critical transition between pre- and post-implantation are hampered by the inaccessibility of relevant tissues for study or experimental manipulation. It has been a long-standing goal to recapitulate this process in vitro.

When pluripotent stem cells are cultured in suspension in the absence of pluripotency-supporting cytokines, they spontaneously aggregate into spherical embryoid bodies (EBs), eventually undergoing cavitation. For decades, EBs have served as an extremely valuable tool for recapitulating early events of embryonic differentiation [3]. However, there are several known limitations to this model system. EB cultures do not follow the spatiotemporal events of embryogenesis and lack the organization found in gastrulating embryos. Additionally, EB cultures typically start with several hundred cells, whereas implantation-stage blastocysts contain on average $8-16$ epiblast cells [4]. These shortcomings of the model system led to a misconception concerning cavitation during embryogenesis. Until recently, it was widely accepted that the pro-amniotic cavity in post-implantation mouse embryos is formed through apoptosis, the underlying mechanism of EB cavity formation. Instead, we now understand that blastocyst cavitation is formed by apical cellular constriction, which is hypothesized to be a result of polarization events initiated by primitive endodermand trophectoderm-secreted factors [5]. The current study by Zernicka-Goetz and colleagues builds upon this previously established hypothesis and significantly enhances the EB model system by combining a single embryonic stem cell together with a small clump of trophoblast stem cells, generating a structure termed ETS-embryos. Upon aggregation in an extra-cellular matrix scaffold (Matrigel), the cells undergo spontaneous patterning and lumeno- genesis to form an elongated cylindrical shape, remarkably similar to natural embryos. Surprisingly and in stark contrast to EBs, ETS-embryo development closely mimics post-implantation embryos in cell numbers and spatiotemporal dynamics [6].

Making use of their new ETSembryo model system, the authors observed five morphogenetic events between implantation and germ layer specification with dynamics comparable to natural embryos. These steps involve spontaneous self-organization into rosette-like configuration, leading to polarization and subsequent epithelization and lumenogenesis in the embryonic compartment followed by the extra-embryonic compartment. Next, both cavities fuse into a structure resembling the pro-amniotic cavity. Then there emerges a characteristic embryo structure induced by Nodal signaling, followed by symmetry breaking and Wnt-dependent localized induction of mesoderm markers. Finally, BMP signals induce specification of primordial germ cell (PGC)-like cells at the ESCTSC boundary [6]. A goal of future studies will be to elucidate the mechanism of the symmetry breaking event during mesoderm specification in the absence of primitive endoderm.

While attempts to co-culture ESCs with TSCs were pursued by numerous groups soon after the first TSC lines were reported, it is remarkable that it took almost 20 years since TSCs were derived before the successful generation of ETS-embryos was finally achieved. This emphasizes the importance of establishing the right medium formulation which allows co-development 
of ESCs and TSCs. Further, providing an extra-cellular matrix scaffold seems critical. In fact, the authors hypothesize that Matrigel substituted for primitive endoderm to provide necessary cues for proper embryo development [6]. The latter suggests an urgent need to reevaluate our current cell culture models, emphasizing that cells do not function as isolated entities but instead are part of a complex network in which they strongly depend on cell-cell communication.

Due to the infinite supply of cultured stem cells, their successful assembly into ETS-embryos offers a powerful tool to study embryogenesis outside of the reproductive tract, making it readily accessible for pathway modification and identification of the underlying mechanisms. Consequently, the intriguing next step will be to apply this system to human embryonic and extra-embryonic cells. Combined with recent advances in the in vitro culture of human embryos beyond implantation stage [7, 8], ETSembryos would hold great promise for eliminating the need for human embryos for research purposes. Shortage of embryos available for research along with the ethical concerns regarding their use has been a major obstacle to advancing our understanding of basic mechanisms in human physiological and pathological development. However, as defined by the International Society for Stem Cell Research Guidelines for Stem Cell Research and Clinical Translation [9], experiments to reassemble human stem cells into embryo-like structures should also trigger independent ethical review, and may warrant a re-evaluation of the

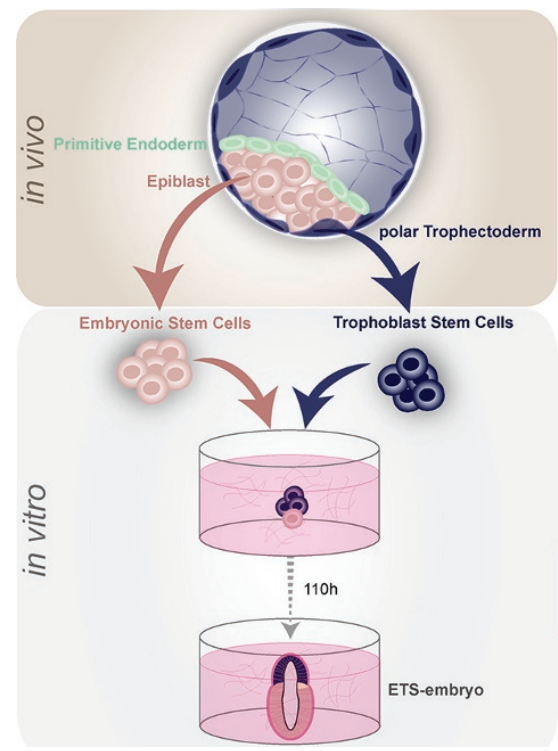

Figure 1 Blastocyst stage embryos comprise pluripotent epiblasts (pink) and multipotent trophoblast stem cells (blue), which can be cultured indefinitely in vitro. Upon re-aggregation in a Matrigel-scaffold and medium conditions supporting co-development of both stem cell types, these cells self-assemble into an artificial embryonic architecture closely resembling egg-cylinder stage embryos. Induction of primordial germ cell-like cells (yellow) is initiated at the boarder of the trophoblast and embryonic compartment.

14-day rule, which specifies temporal and anatomic limits on the types of studies that can be performed on human embryos, and by extension, embryo-like structures should they manifest human developmental potential. Currently a major barrier to the establishment of human ETS-embryos is the lack of bona fide human TSC lines. Despite several attempts to derive these cells from blastocysts and placental tissue of various gestational stages, human TSCs remain elusive [10]. If this hurdle could be overcome, ETS-embryos might shed light onto critical milestones of embryogenesis and help to understand why such a significant number of pregnancies fail prior to or during implantation. Addressing unmet medical needs might provide compelling justification for further exploration of ETS-embryos.

\section{Caroline Kubaczka ${ }^{1,2,3}$, George Q Daley ${ }^{1,2,3}$}

${ }^{1}$ Stem Cell Transplantation Program, Division of Pediatric Hematology and Oncology, Boston Children's Hospital and Dana-Farber Cancer Institute, Boston, MA 02115, USA; ${ }^{2}$ Department of Biological Chemistry and Molecular Pharmacology, Harvard Medical School, Boston, MA 02115, USA; ${ }^{3}$ Harvard Stem Cell Institute, Cambridge, MA 02138, USA

Correspondence: George Q Daley

E-mail: George_Daley@hms.harvard.edu

\section{References}

1 Evans MJ, Kaufman MH. Nature 1981; 292:154-156.

2 Tanaka S, Kunath T, Hadjantonakis AK, et al. Science 1998; 282:2072-2075.

3 Martin GR, Evans MJ. Proc Natl Acad Sci USA 1975; 72:1441-1445.

4 Morris SA, Guo Y, Zernicka-Goetz M. Cell Rep 2012; 2:756-765.

5 Bedzhov I, Zernicka-Goetz M. Cell 2014; 156:1032-1044.

6 Harrison SE, Sozen B, Christodoulou N, et al. Science 2017; 356: pii: eaal1810.

7 Deglincerti A, Croft GF, Pietila LN, et al. Nature 2016; 533:251-254.

8 Shahbazi MN, Jedrusik A, Vuoristo S, et al. Nat Cell Biol 2016; 18:700-708.

9 Daley GQ, Hyun I, Apperley JF, et al. Stem Cell Reports 2016; 6:787-797.

10 Chang CW, Parast MM. Placenta 2017 Jan 5. doi: 10.1016/j.placenta.2017.01.003. 